\title{
Best Proximity Point Theorems for a Berinde MT-Cyclic Contraction on a Semisharp Proximal Pair
}

\author{
Chalongchai Klanarong ${ }^{1}$ and Tanadon Chaobankoh $\mathbb{D D}^{2}$ \\ ${ }^{1}$ Department of Mathematics, Faculty of Science, Mahasarakham University, Mahasarakham 44150, Thailand \\ ${ }^{2}$ Center of Excellence in Mathematics and Applied Mathematics, Department of Mathematics, Faculty of Science, \\ Chiang Mai University, Chiang Mai 50200, Thailand
}

Correspondence should be addressed to Tanadon Chaobankoh; tanadon.c@cmu.ac.th

Received 22 June 2018; Revised 24 September 2018; Accepted 14 October 2018; Published 30 October 2018

Academic Editor: Vladimir V. Mityushev

Copyright (c) 2018 Chalongchai Klanarong and Tanadon Chaobankoh. This is an open access article distributed under the Creative Commons Attribution License, which permits unrestricted use, distribution, and reproduction in any medium, provided the original work is properly cited.

In this paper, a new type of non-self-mapping, called Berinde MT-cyclic contractions, is introduced and studied. Best proximity point theorems for this type of mappings in a metric space are presented. Some examples illustrating our main results are also given. Our results generalize and improve some known results in the literature.

\section{Introduction and Preliminaries}

Several problems in a real world can be modeled in the form of operator equations. An equation $S x=x$, which is called the fixed point equation, is one of the important means for solving some problems among them. Fixed point theory is then considered as an important tool for solving such problems. The well-known fixed point theorem for contraction mappings was given by Banach [1]. It is known as the Banach contraction principle. This principle guarantees that each contraction mapping from a complete metric space $X$ into itself always has a unique fixed point.

In 2004, Berinde [2] introduced and studied the concept of weak contraction mappings in the context of a complete metric space. Let $(X, d)$ be a metric space. A mapping $S$ : $X \longrightarrow X$ is called a weak contraction if there exist $a \in(0,1)$ and $L \geq 0$ such that

$$
d(S x, S y) \leq a d(x, y)+L d(x, S y), \quad \text { for all } x, y \in X
$$

A fixed point theorem of this type of mapping was proved in [2]. It extended and generalized that of the Banach contraction principle and others; see [2] and references therein.

On the other hand, if the fixed point equation $S x=x$ does not have a solution, then $d(x, S x)>0$ for all $x \in X$.
In this situation, it is natural to ask whether we can find an approximate solution $x$ such that the error is

$$
d(x, S x)=\min _{y \in X} d(y, S y) .
$$

In order to have a concrete lower bound, let us consider two nonempty subsets $W$ and $V$ of a metric space $X$ and a mapping $S: W \longrightarrow V$. It is observed that $d(x, S x) \geq d(W, V)$ for all $x \in W$, where $d(W, V)=\inf \{d(x, y): x \in W$ and $y \in$ $V\}$. So we are interested to find a point $x_{0} \in W$ such that

$$
d\left(x_{0}, S x_{0}\right)=d(W, V) .
$$

Such point $x_{0}$ is called a best proximity point of the mapping $S$, and $d\left(x_{0}, S x_{0}\right)$ is called the global minimum value of $d(W, V)$.

The best proximity point theorem was first studied by Fan [3], in 1969. He proved that if $W$ is a nonempty compact convex subset in a normed space $X$ and $S: W \longrightarrow X$ is a continuous mapping, then there exists $u \in W$ such that $\|u-S u\|=d(S u, W)$ where $d(S u, W):=\min \{\|S u-a\|: a \epsilon$ $W$ \}. Especially, if $S(W) \subseteq W$, then we get that $u$ is a fixed point of $S$.

Several years later, the above result has been studied and generalized by many researchers, such as Reich [4], Sehgal and Singh [5], Vetrivel et al. [6], Anuradha and Veeramani [7], Basha [8, 9], Kirk et al. [10], Raj [11], Gabeleh [12], Abkar 
and Gabeleh [13], Eldred and Veeramani [14], and Du and Lakzian [15] and references therein. Some recent research papers worth mentioning are [16-19].

Throughout this paper, we denote by $W$ and $V$ nonempty subsets of a metric space $(X, d)$. We also require the following notions:

$$
\begin{aligned}
& d(W, V):=\inf \{d(x, y): x \in W \text { and } y \in V\}, \\
& W_{0}:=\{x \in W: d(x, y)=d(W, V) \text { for some } y \in V\}, \\
& V_{0}:=\{y \in V: d(x, y)=d(W, V) \text { for some } x \in W\} .
\end{aligned}
$$

A mapping $S: W \cup V \longrightarrow W \cup V$ is called a cyclic mapping if $S(W) \subseteq V$ and $S(V) \subseteq W$. And a point $x \in W \cup V$ is said to be a best proximity point of $S$ if $d(x, S x)=d(W, V)$. In 2006, Eldred and Veeramani [14] introduced the concept of cyclic contraction and proved the existence of a best proximity point for this type of mapping on a complete metric space.

Definition 1 (see [14]). A mapping $S: W \cup V \longrightarrow W \cup V$ is called a cyclic contraction if the following conditions hold:

\section{(i) $S$ is a cyclic mapping;}

(ii) there exists $k \in(0,1)$ such that $d(S x, S y) \leq k d(x, y)+$ $(1-k) d(W, V)$ for all $x \in W, y \in V$.

The concept of MT-function was used by Reich [20] and Mizoguchi and Takahashi [21] to define a class of multivalued mappings which is more general than that of contraction mappings. After that Du $[22,23]$ studied the class of multivalued mappings generated by Mizoguchi and Takahashi functions (or MT-functions) and gave characterizations of MT-functions.

Definition 2 (see [22]). A function $\varphi:[0, \infty) \longrightarrow[0,1)$ is said to be an MT-function (or R-function) if $\limsup _{s \rightarrow t^{+}} \varphi(s)<1$ for all $t \in[0, \infty)$.

Theorem 3 (see [23]). Let $\varphi:[0, \infty) \longrightarrow[0,1)$ be a function. Then the following statements are equivalent.

(a) $\varphi$ is an MT-function; i.e., $\lim \sup _{s \rightarrow t^{+}} \varphi(s)<1$ for all $t \in[0, \infty)$.

(b) For each $t \in[0, \infty)$, there exist $r_{t} \in[0,1)$ and $\varepsilon_{t}>0$ such that $\varphi(s) \leq r_{t}$ for all $s \in\left(t, t+\varepsilon_{t}\right)$.

(c) For any nonincreasing sequence $\left\{x_{n}\right\}_{n \in \mathbb{N}}$ in $[0, \infty), 0 \leq$ $\sup _{n \in \mathbb{N}} \varphi\left(x_{n}\right)<1$.

It is clear that if $\varphi$ is a nondecreasing function or a nonincreasing function, then $\varphi$ is an MT-function. For more examples and details, see $[15,22,23]$.

Consequently, Du and Lakzian [15] introduced MT-cyclic contractions with respect to $\varphi$ and proved the existence and convergence theorems for this type of non-self-mapping in metric spaces.

Definition 4 (see [15]). If a map $S: W \cup V \longrightarrow W \cup V$ satisfies the following: then $S$ is called an MT-cyclic contraction with respect to $\varphi$ on $W \cup V$. (i) $S$ is a cyclic mapping;

(ii) there exists an MT-function $\varphi:[0, \infty) \rightarrow[0,1)$ such that $d(S x, S y) \leq \varphi(d(x, y)) d(x, y)+(1-$ $\varphi(d(x, y))) d(W, V)$ for all $x \in W$ and $y \in V$.

It is obvious that if $\varphi(t)=k$ with $k \in[0,1)$, then $S$ is a cyclic contraction, and, hence, an MT-cyclic contraction with respect to $\varphi$ which is more general than that of cyclic contraction. For example of an MT-cyclic contraction with respect to $\varphi$, but is not a cyclic contraction, see [15].

In 2009, Suzuki et al. [24] introduced the concept of the property UC of two nonempty subsets of a metric space as follows.

Definition 5 (see [24]). Let $(W, V)$ be a pair of nonempty subsets of a metric space $(X, d)$. The pair $(W, V)$ is said to satisfy the property $U C$ if $\left\{x_{n}\right\}$ and $\left\{z_{n}\right\}$ are sequences in $W$ and $\left\{y_{n}\right\}$ is a sequence in $V$ such that

$$
\lim _{n \longrightarrow \infty} d\left(x_{n}, y_{n}\right)=d(W, V)=\lim _{n \longrightarrow \infty} d\left(z_{n}, y_{n}\right),
$$

and then $\lim _{n \rightarrow \infty} d\left(x_{n}, z_{n}\right)=0$.

Later, in 2011, Kosuru and Veeramani [25] introduced the concept of semisharp proximal pair of two nonempty subsets of a metric space. This concept is again more general than that of the property UC.

Definition 6 (see [25]). Let $(W, V)$ be a pair of nonempty subsets of a metric space $(X, d)$. The pair $(W, V)$ is said to be a semisharp proximal pair if for each $w \in W$ and $u \in V$ there exist at most one $x \in V$ and $y \in W$ such that $d(w, x)=$ $d(W, V)=d(y, u)$.

Example 7. Consider the space $X$ of all real valued continuous functions on $[0,1]$ with the supremum norm; i.e., $X=$ $\left(C[0,1],\|\cdot\|_{\infty}\right)$. Set

$$
\begin{aligned}
W & =\left\{f_{t}: t \in[0,1]\right\} \\
\text { and } V & =\left\{g_{t}: t \in[0,1]\right\},
\end{aligned}
$$

where

$$
f_{t}(x)= \begin{cases}t+x, & \text { if } x \in\left[0, \frac{1}{2}\right] ; \\ t-x+1, & \text { if } x \in\left[\frac{1}{2}, 1\right],\end{cases}
$$

and

$$
g_{t}(x)= \begin{cases}t+x+1, & \text { if } x \in\left[0, \frac{1}{2}\right] ; \\ t-x+2, & \text { if } x \in\left[\frac{1}{2}, 1\right]\end{cases}
$$

It is easy to show that $\left\|f_{t}-g_{t}\right\|=1$ and $\left\|f_{t}-g_{k}\right\|>1$ for all $t \neq k \in[0,1]$. Hence $d(W, V)=1$ and $(W, V)$ is a semisharp proximal pair.

Note that the property UC implies semisharp proximality. In 2015, R. Espinola, et al. [26] introduced the concept of 
a proximally complete pair $(W, V)$ of subsets of a metric space. They proved existences and convergence theorems of best proximity points for cyclic contraction mappings. They obtained a useful theorem presented as follows.

Theorem 8 (Espinola et al. [26, Theorem 3.3]). Let $(W, V)$ be a pair of complete subsets of a metric space satisfying the UC property. If $\left\{x_{n}\right\}$ is a sequence in $W \cup V$ with $x_{2 n} \in W$ and $x_{2 n+1} \in V$, for all $n \in \mathbb{N}$, then the sequences $\left\{x_{2 n}\right\}$ and $\left\{x_{2 n+1}\right\}$ have convergent subsequences in $W$ and $V$, respectively.

By those works mentioned above, we aim to introduce a new type of single-valued, non-self-mapping which is more general than that of contractions, cyclic contractions, and $M T$-cyclic contractions. The best proximity point theorems for this type of mappings in metric spaces will be investigated. Our main results extend and generalize those of $\mathrm{Du}$ and Lakzian [15], Eldred and Veeramani [14], and others.

\section{Main Results}

By using ideas of cyclic contractions, MT-functions, and weak contractions, we shall first introduce Berinde MT-cyclic contractions with respect to $\varphi$ and prove the existence and convergence theorems for this type of non-self-mapping in metric spaces.

Definition 9. Let $S: W \cup V \longrightarrow W \cup V$ be a cyclic mapping. The mapping $S$ is said to be a Berinde MT-cyclic contraction with respect to $\varphi$ if there exists an MT-function $\varphi:[0, \infty) \longrightarrow$ $[0,1)$ and $L \geq 0$ such that

$$
\begin{aligned}
d(S x, S y) \leq & \varphi(d(x, y)) d(x, y) \\
& +(1-\varphi(d(x, y))) d(W, V) \\
& +L \min \{d(y, S x), d(x, S y)\},
\end{aligned}
$$

for all $x \in W$ and $y \in V$.

It is easy to see that a Berinde MT-cyclic contraction with respect to $\varphi$ can be reduced to an MT-cyclic contraction with respect to $\varphi$.

Remark 10. If $S: W \cup V \longrightarrow W \cup V$ is a Berinde MT-cyclic contraction with respect to $\varphi$, then $S$ satisfies the following condition:

$$
d(S x, S y) \leq d(x, y)+L \min \{d(y, S x), d(x, S y)\},
$$

for all $x \in W$ and $y \in V$. To see this, we can write (9) in the form $d(S x, S y)-d(W, V) \leq \varphi(d(x, y))[d(x, y)-d(W, V)]+$ $L \min \{d(y, S x), d(x, S y)\}$, for all $x \in W$ and $y \in V$. Because of $\varphi(d(x, y))<1$, it follows that

$$
\begin{aligned}
& d(S x, S y)-d(W, V) \\
& \leq d(x, y)-d(W, V) \\
& \quad+L \min \{d(y, S x), d(x, S y)\},
\end{aligned}
$$

and, for all $x \in W$ and $y \in V$, hence (10) is satisfied.
Example 11. Let $l^{\infty}$ be the metric space consisting of all bounded real sequences with supremum metric $d_{\infty}$ and let $\left\{e_{n}\right\}$ be the canonical basis of $c_{0}$, where $c_{0}$ is the space of all null sequences. Let $\left\{\tau_{n}\right\}$ be a sequence of positive real numbers satisfying $\tau_{1}=\tau_{2}$ and $0<a \leq \tau_{n+1}<\tau_{n}$ for $n \geq 2$ and for some positive real number $a$. Thus $\left\{\tau_{n}\right\}$ is convergent. Put $x_{n}=\tau_{n} e_{n}$ for $n \in \mathbb{N}$ and let $X=\left\{x_{1}, x_{2}, x_{3}, \ldots\right\}$. Then $X$ is a bounded and complete subset of $l^{\infty}$, and hence $\left(X, d_{\infty}\right)$ is a complete metric space with $d_{\infty}\left(x_{n}, x_{m}\right)=\tau_{n}$ if $m>n$.

Let $W=\left\{x_{1}, x_{3}, x_{5}, \ldots\right\}, V=\left\{x_{1}, x_{2}, x_{4}, \ldots\right\}$ and let $S$ : $W \cup V \longrightarrow W \cup V$ be defined by

$$
S x_{n}= \begin{cases}x_{1} & \text { if } n=1,2 \\ x_{n+3} & \text { if } n \geq 3\end{cases}
$$

and define $\varphi:[0, \infty) \longrightarrow[0,1)$ by

$$
\begin{aligned}
& \varphi(t) \\
& = \begin{cases}\frac{\tau_{n+3}}{\tau_{n}} & \text { if } t=\tau_{n} \text { for some } n \in \mathbb{N} \text { with } n \geq 3 ; \\
0 & \text { otherwise. }\end{cases}
\end{aligned}
$$

Then $d(W, V)=0$ and $S(W) \subseteq V, S(V) \subseteq W$. Since $\limsup _{s \rightarrow t^{+}} \varphi(s)=0<1$ for all $t \in[0, \infty)$, we have that $\varphi$ is an $M T$-function. Next, we show that $S$ is a Berinde $M T$ cyclic contraction with respect to $\varphi$. Obviously, $d\left(S x_{1}, S x_{i}\right)$ for $i=1,2,3, \ldots$ satisfy (9) with $L \geq 1$. We will consider three cases as follows.

Case 1. For $i \geq 3$ and $x_{i} \in W$, we have

$$
\begin{aligned}
d\left(S x_{2}, S x_{i}\right)= & d\left(x_{1}, x_{i+3}\right)=\tau_{1} \leq L \tau_{2} \\
= & \varphi\left(d\left(x_{2}, x_{i}\right)\right) d\left(x_{2}, x_{i}\right) \\
& +\left(1-\varphi\left(d\left(x_{2}, x_{i}\right)\right)\right) d(W, V) \\
& +L \min \left\{d\left(x_{2}, x_{i+3}\right), d\left(x_{i}, x_{1}\right)\right\},
\end{aligned}
$$

for $L \geq 1$.

Case 2. For $i \geq 4$ and $x_{i} \in V$, we get

$$
\begin{aligned}
d\left(S x_{3}, S x_{i}\right)= & d\left(x_{6}, x_{i+3}\right)=\tau_{6} \\
\leq & \tau_{6}+L \min \left\{d\left(x_{3}, x_{i+3}\right), d\left(x_{i}, x_{6}\right)\right\} \\
= & \varphi\left(d\left(x_{3}, x_{i}\right)\right) d\left(x_{3}, x_{i}\right) \\
& +\left(1-\varphi\left(d\left(x_{3}, x_{i}\right)\right)\right) d(W, V) \\
& +L \min \left\{d\left(x_{3}, x_{i+3}\right), d\left(x_{i}, x_{6}\right)\right\}
\end{aligned}
$$

for $L \geq 0$. 
Case 3. For $j>i \geq 4$ and $x_{i}, x_{j} \in W \cup V$, we have

$$
\begin{aligned}
d\left(S x_{i}, S x_{j}\right)= & d\left(x_{i+3}, x_{j+3}\right)=\tau_{i+3} \\
\leq & \tau_{i+3} \\
& +L \min \left\{d\left(x_{i}, x_{j+3}\right), d\left(x_{j}, x_{i+3}\right)\right\} \\
= & \varphi\left(d\left(x_{i}, x_{j}\right)\right) d\left(x_{i}, x_{j}\right) \\
& +\left(1-\varphi\left(d\left(x_{i}, x_{j}\right)\right)\right) d(W, V) \\
& +L \min \left\{d\left(x_{i}, x_{j+3}\right), d\left(x_{j}, x_{i+3}\right)\right\},
\end{aligned}
$$

for $L \geq 0$.

From all of the above cases, we can now conclude that $S$ is a Berinde $M T$-cyclic contraction with respect to $\varphi$ and $L \geq 1$. We note from Case 1 that $S$ is not an $M T$-cyclic contraction with respect to $\varphi$.

Proposition 12. Let $W$ and $V$ be nonempty subsets of a metric space $(X, d)$ and $S: W \cup V \longrightarrow W \cup V$ be a Berinde MT-cyclic contraction with respect to $\varphi$. Then starting with any given $x_{0} \in$ $W \cup V$, define a sequence $\left\{x_{n}\right\}$ in $W \cup V$ by $x_{n+1}=S x_{n}$ for all $n \geq 0$; we have $d\left(x_{n}, x_{n+1}\right) \longrightarrow d(W, V)$ as $n \longrightarrow \infty$.

Proof. Let $x_{0} \in W \cup V$ be given. Define a sequence $\left\{x_{n}\right\}$ in $W \cup V$ by

$$
x_{n+1}=S x_{n}, \quad \text { for each } n \geq 0 .
$$

Suppose that $x_{0} \in W$ (when $x_{0} \in V$ is similar); then $x_{1}=$ $S x_{0} \in V$, and so, $x_{2}=S x_{1} \in W$. Since $S$ is a Berinde MTcyclic contraction with respect to $\varphi$, we have

$$
\begin{aligned}
d\left(x_{1}, x_{2}\right)= & d\left(S x_{0}, S x_{1}\right) \\
\leq & \varphi\left(d\left(x_{0}, x_{1}\right)\right) d\left(x_{0}, x_{1}\right) \\
& +\left(1-\varphi\left(d\left(x_{0}, x_{1}\right)\right)\right) d(W, V) \\
& +L \min \left\{d\left(x_{1}, S x_{0}\right), d\left(x_{0}, S x_{1}\right)\right\} \\
= & \varphi\left(d\left(x_{0}, x_{1}\right)\right) d\left(x_{0}, x_{1}\right) \\
& +\left(1-\varphi\left(d\left(x_{0}, x_{1}\right)\right)\right) d(W, V) \\
& +L \min \left\{d\left(x_{1}, x_{1}\right), d\left(x_{0}, x_{2}\right)\right\} \\
= & \varphi\left(d\left(x_{0}, x_{1}\right)\right) d\left(x_{0}, x_{1}\right) \\
& +\left(1-\varphi\left(d\left(x_{0}, x_{1}\right)\right)\right) d(W, V) .
\end{aligned}
$$

Again, since $x_{2} \in W$ and $S$ is a cyclic mapping, we get $x_{3}=$ $S x_{2} \in V$. By the Berinde MT-cyclic contraction with respect to $\varphi$ of $S$, we have

$$
\begin{aligned}
d\left(x_{2}, x_{3}\right)= & d\left(S x_{1}, S x_{2}\right) \\
\leq & \varphi\left(d\left(x_{1}, x_{2}\right)\right) d\left(x_{1}, x_{2}\right) \\
& +\left(1-\varphi\left(d\left(x_{1}, x_{2}\right)\right)\right) d(W, V)
\end{aligned}
$$

$$
\begin{aligned}
& +L \min \left\{d\left(x_{1}, S x_{2}\right), d\left(x_{2}, S x_{1}\right)\right\} \\
= & \varphi\left(d\left(x_{1}, x_{2}\right)\right) d\left(x_{1}, x_{2}\right) \\
& +\left(1-\varphi\left(d\left(x_{1}, x_{2}\right)\right)\right) d(W, V) \\
& +L \min \left\{d\left(x_{1}, x_{3}\right), d\left(x_{2}, x_{2}\right)\right\} \\
= & \varphi\left(d\left(x_{1}, x_{2}\right)\right) d\left(x_{1}, x_{2}\right) \\
& +\left(1-\varphi\left(d\left(x_{1}, x_{2}\right)\right)\right) d(W, V) .
\end{aligned}
$$

By induction, we can show that, for each $n \geq 1$,

$$
\begin{aligned}
d\left(x_{n}, x_{n+1}\right) \leq & \varphi\left(d\left(x_{n-1}, x_{n}\right)\right) d\left(x_{n-1}, x_{n}\right) \\
& +\left(1-\varphi\left(d\left(x_{n-1}, x_{n}\right)\right)\right) d(W, V) .
\end{aligned}
$$

By Remark 10, we have

$$
\begin{aligned}
d\left(x_{n}, x_{n+1}\right)= & d\left(S x_{n-1}, S x_{n}\right) \\
\leq & d\left(x_{n-1}, x_{n}\right) \\
& +L \min \left\{d\left(x_{n-1}, S x_{n}\right), d\left(x_{n}, S x_{n-1}\right)\right\} \\
= & d\left(x_{n-1}, x_{n}\right),
\end{aligned}
$$

for all $n \geq 1$. It means that $\left\{d\left(x_{n}, x_{n+1}\right)\right\}$ is a nonincreasing sequence. By Theorem 3 , we get $0 \leq \sup _{n \in \mathbb{N}} \varphi\left(d\left(x_{n}, x_{n+1}\right)\right)<$ 1.

Put $k:=\sup _{n \in \mathbb{N}} \varphi\left(d\left(x_{n}, x_{n+1}\right)\right)$. Thus $0 \leq \varphi\left(d\left(x_{n}, x_{n+1}\right)\right) \leq$ $k<1$, for all $n \geq 1$. It follows from (20) that

$$
d\left(x_{n}, x_{n+1}\right) \leq k d\left(x_{n-1}, x_{n}\right)+(1-k) d(W, V),
$$
for all $n \geq 1$.

Hence for each $n \geq 1$, we have

$$
\begin{gathered}
d\left(x_{n}, x_{n+1}\right) \leq k d\left(x_{n-1}, x_{n}\right)+(1-k) d(W, V) \\
\leq k\left[k\left(d\left(x_{n-2}, x_{n-1}\right)+(1-k) d(W, V)\right)\right] \\
\quad+(1-k) d(W, V) \\
=k^{2}\left(d\left(x_{n-2}, x_{n-1}\right)+\left(1-k^{2}\right) d(W, V)\right) \\
\vdots \\
\leq k^{n}\left(d\left(x_{0}, x_{1}\right)+\left(1-k^{n}\right) d(W, V)\right) .
\end{gathered}
$$

Since $d(W, V) \leq d\left(x_{n}, x_{n+1}\right)$ and $k^{n} \longrightarrow 0$ as $n \longrightarrow \infty$, by taking $n \longrightarrow \infty$ in the above inequality, we obtain that

$$
d\left(x_{n}, x_{n+1}\right) \longrightarrow d(W, V), \quad \text { as } n \longrightarrow \infty .
$$

The proof is now completed.

Example 13. Let $\tau_{n}=1 / 3+1 / n$ for all $n \in \mathbb{N}$. Then $\lim _{n \rightarrow \infty} \tau_{n}=1 / 3$. Put $x_{n}=\tau_{n} e_{n}$ for $n \in \mathbb{N}$ and let $X=$ $\left\{x_{1}, x_{2}, x_{3}, \ldots\right\}$ be a bounded and complete subset of $l^{\infty}$. Then 
$\left(X, d_{\infty}\right)$ be a complete metric space with $d_{\infty}\left(x_{n}, x_{m}\right)=\tau_{n}$ if $m>n$. Set $W=\left\{x_{1}, x_{3}, x_{5}, \ldots\right\}$ and $V=\left\{x_{2}, x_{4}, x_{6}, \ldots\right\}$. So $\lim _{n \rightarrow \infty} d\left(x_{n}, x_{n+1}\right)=\lim _{n \rightarrow \infty} \tau_{n}=1 / 3=d(W, V)$. Let $S: W \cap V \longrightarrow W \cap V$ be defined by

$$
S x_{n}=x_{n+1} \quad \text { for all } n \in \mathbb{N} \text {. }
$$

It is easy to see that $S(W)=V$ and $S(V) \subset W$ and so $S$ is a cyclic mapping. Define $\varphi:[0, \infty) \longrightarrow[0,1)$ as

$$
\varphi(t)= \begin{cases}\frac{\tau_{n+1}}{\tau_{n}} & \text { if } t=\tau_{n} \text { for some } n \in \mathbb{N} \\ 0 & \text { otherwise. }\end{cases}
$$

Then $\varphi$ is an MT-function. Now, we will show that $S$ is a Berinde MT-cyclic contraction with respect to $\varphi$. For $m, n \in$ $\mathbb{N}$ with $m>n$,

$$
\begin{aligned}
d\left(S x_{n}, S x_{m}\right)= & \tau_{n+1} \\
< & \tau_{n+1}+\frac{1}{3}\left(1-\frac{\tau_{n+1}}{\tau_{n}}\right) \\
& +L \min \left\{d\left(x_{n}, S x_{m}\right), d\left(x_{m}, S x_{n}\right)\right\} \\
= & \varphi\left(d\left(x_{n}, x_{m}\right)\right) d\left(x_{n}, x_{m}\right) \\
& +\left(1-\varphi\left(d\left(x_{n}, x_{m}\right)\right)\right) d(W, V) \\
& +L \min \left\{d\left(x_{n}, S x_{m}\right), d\left(x_{m}, S x_{n}\right)\right\}
\end{aligned}
$$

Hence $S$ is a Berinde MT-cyclic contraction with respect to $\varphi$. Therefore, all the assumptions of Proposition 12 hold.

The following result is obtained immediately from Proposition 12 because every nondecreasing function or nonincreasing function is an MT-function.

Corollary 14. Let $W$ and $V$ be nonempty subsets of a metric space $(X, d)$ and $S: W \cup V \longrightarrow W \cup V$ be a cyclic mapping. Let $x_{0} \in W$ be given and define a sequence $\left\{x_{n}\right\}$ in $W \cup V$ by $x_{n+1}=$ $S x_{n}$, for all $n \geq 0$. Suppose that there exists a nondecreasing (or nonincreasing) function $\eta:[0, \infty) \longrightarrow[0,1)$ such that for all $x \in W$ and $y \in V$

$$
\begin{aligned}
d(S x, S y) \leq & \eta(d(x, y)) d(x, y) \\
& +(1-\eta(d(x, y))) d(W, V) \\
& +L \min \{d(y, S x), d(x, S y)\} .
\end{aligned}
$$

Then $\lim _{n \rightarrow \infty} d\left(x_{n}, x_{n+1}\right)=d(W, V)$.

By Proposition 12, if $S$ is a cyclic contraction or an MT-cyclic contraction with respect to $\varphi$, then we obtain directly the following results which were proved by Eldred and Veeramani [14] and Du and Lakzian [15], respectively.

Corollary 15 (Eldred and Veeramani [14, Proposition 3.1]). Let $W$ and $V$ be nonempty subsets of a metric space $(X, d)$ and $S: W \cup V \longrightarrow W \cup V$ be a cyclic contraction. Then starting with any $x_{0} \in W$ and defining a sequence $\left\{x_{n}\right\}$ in $W \cup V$ by $x_{n+1}=S x_{n}$, for all $n \geq 0$, then $\lim _{n \rightarrow \infty} d\left(x_{n}, x_{n+1}\right)=d(W, V)$.
Corollary 16 (Du and Lakzian [15, Theorem 2.1]). Let $W$ and $V$ be nonempty subsets of a metric space $(X, d)$ and $S: W \cup$ $V \longrightarrow W \cup V$ be an MT-cyclic contraction with respect to $\varphi$. Then starting with any $x_{0} \in W \cup V$, define a sequence $\left\{x_{n}\right\}$ in $W \cup V$ by $x_{n+1}=S x_{n}$ for all $n \geq 0$; we have $d\left(x_{n}, x_{n+1}\right) \longrightarrow$ $d(W, V)$ as $n \longrightarrow \infty$.

Observe that if $W$ and $V$ are nonempty subsets of a metric space $(X, d)$ and $S: W \cup V \longrightarrow W \cup V$ is a cyclic mapping with $x_{0} \in W$, define a sequence $\left\{x_{n}\right\}$ in $W \cup V$ by $x_{n+1}=S x_{n}$, for all $n \geq 0$; then $\left\{x_{2 n}\right\}$ and $\left\{x_{2 n+1}\right\}$ are subsequences of $\left\{x_{n}\right\}$ in $W$ and $V$, respectively. Similarly, if $x_{0} \in V$, then $\left\{x_{2 n}\right\}$ and $\left\{x_{2 n+1}\right\}$ are subsequences of $\left\{x_{n}\right\}$ in $V$ and $W$, respectively. Moreover, $d(W, V) \leq d\left(x_{n}, x_{n+1}\right)$, for all $n \geq 0$.

Theorem 17. Let $W$ and $V$ be nonempty subsets of a metric space $(X, d)$ and $S: W \cup V \longrightarrow W \cup V$ be a cyclic mapping. Let $x_{0} \in W$ be given and define a sequence $\left\{x_{n}\right\}$ in $W \cup V$ by $x_{n+1}=S x_{n}$, for all $n \geq 0$. Suppose that the following conditions hold:

(i) $d(S x, S y) \leq d(x, y)+L \min \{d(x, S y), d(y, S x)\}$ for all $x \in W$ and $y \in V$ with $L \geq 0$;

(ii) $\left\{x_{2 n}\right\}$ has a convergent subsequence in $W$;

(iii) $\lim _{n \longrightarrow \infty} d\left(x_{n}, x_{n+1}\right)=d(W, V)$.

Then there exists $x \in W$ such that $d(x, S x)=d(W, V)$.

Proof. Let $x_{0} \in W$ and $\left\{x_{2 n_{k}}\right\}$ be a subsequence of $\left\{x_{2 n}\right\}$ such that

$$
\lim _{k \rightarrow \infty} x_{2 n_{k}}=x, \quad \text { for some } x \in W \text {. }
$$

In fact, for each $k \in \mathbb{N}$,

$$
\begin{aligned}
d(W, V) & \leq d\left(x, x_{2 n_{k}-1}\right) \\
& \leq d\left(x, x_{2 n_{k}}\right)+d\left(x_{2 n_{k}}, x_{2 n_{k}-1}\right) .
\end{aligned}
$$

It follows by (iii) that

$$
\lim _{k \rightarrow \infty} d\left(x, x_{2 n_{k}-1}\right)=d(W, V), \quad \text { as } k \longrightarrow \infty .
$$

From $(i)$, for each $k \in \mathbb{N}$, we have

$$
\begin{aligned}
d(W, V) \leq & d\left(S x, x_{2 n_{k}}\right)=d\left(S x, S x_{2 n_{k}-1}\right) \\
\leq & d\left(x, x_{2 n_{k}-1}\right) \\
& +L \min \left\{d\left(x, x_{2 n_{k}}\right), d\left(x_{2 n_{k}-1}, S x\right)\right\} \\
\leq & d\left(x, x_{2 n_{k}-1}\right)+\operatorname{Ld}\left(x, x_{2 n_{k}}\right) .
\end{aligned}
$$

Taking $k \longrightarrow \infty$ in the above inequality, we obtain that

$$
d(x, S x)=\lim _{k \rightarrow \infty} d\left(x_{2 n_{k}}, S x\right)=d(W, V) .
$$

The proof is completed.

Using the same proof as Theorem 17, we obtain a similar result. 
Theorem 18. Let $W$ and $V$ be nonempty subsets of a metric space $(X, d)$ and $S: W \cup V \longrightarrow W \cup V$ be a cyclic mapping. Let $x_{0} \in W$ be given and define a sequence $\left\{x_{n}\right\}$ in $W \cup V$ by $x_{n+1}=S x_{n}$, for all $n \geq 0$. Suppose that the following conditions hold:

(i) $d(S x, S y) \leq d(x, y)+L \min \{d(x, S y), d(y, S x)\}$ for all $x \in W$ and $y \in V$ with $L \geq 0$;

(ii) $\left\{x_{2 n+1}\right\}$ has a convergent subsequence in $V$;

(iii) $\lim _{n \rightarrow \infty} d\left(x_{n}, x_{n+1}\right)=d(W, V)$.

Then there exists $y \in V$ such that $d(y, S y)=d(W, V)$.

Applying Proposition 12 and Theorems 17 and 18, we establish the following new best proximity point theorems for a Berinde MT-cyclic contraction with respect to $\varphi$.

Theorem 19. Let $W$ and $V$ be nonempty subsets of a metric space $(X, d)$ and $S: W \cup V \longrightarrow W \cup V$ be a Berinde MT-cyclic contraction with respect to $\varphi$. Let $x_{0} \in W$ be given and define a sequence $\left\{x_{n}\right\}$ in $W \cup V$ by $x_{n+1}=S x_{n}$, for all $n \geq 0$. If $\left\{x_{2 n}\right\}$ has a convergent subsequence in $W$, then there exists $x \in W$ such that $d(x, S x)=d(W, V)$.

Theorem 20. Let $W$ and $V$ be nonempty subsets of a metric space $(X, d)$ and $S: W \cup V \longrightarrow W \cup V$ be a Berinde MT-cyclic contraction with respect to $\varphi$. Let $x_{0} \in W$ be given and define $a$ sequence $\left\{x_{n}\right\}$ in $W \cup V$ by $x_{n+1}=S x_{n}$, for all $n \geq 0$. If $\left\{x_{2 n+1}\right\}$ has a convergent subsequence in $V$, then there exists $y \in V$ such that $d(y, S y)=d(W, V)$.

By Theorems 19 and 20, we obtain the next result.

Corollary 21. Let $W$ and $V$ be nonempty subsets of a metric space $(X, d)$ and $S: W \cup V \longrightarrow W \cup V$ be a Berinde MT-cyclic contraction with respect to $\varphi$. Let $x_{0} \in W$ be given and define a sequence $\left\{x_{n}\right\}$ in $W \cup V$ by $x_{n+1}=S x_{n}$, for all $n \geq 0$. Suppose that $W$ or $V$ is compact. Then there exists $x \in W \cup V$ such that $d(x, S x)=d(W, V)$.

Theorem 22. Let $W$ and $V$ be nonempty subsets of a metric space $(X, d)$ such that $(W, V)$ is a semisharp proximal pair and $S: W \cup V \longrightarrow W \cup V$ is a Berinde MT-cyclic contraction with respect to $\varphi$. Let $x_{0} \in W$ be given and define a sequence $\left\{x_{n}\right\}$ in $W \cup V$ by $x_{n+1}=S x_{n}$, for all $n \geq 0$. If $\left\{x_{2 n}\right\}$ has a convergent subsequence in $W$, then the following hold:

(i) there exists $x \in W$ such that $d(x, S x)=d(W, V)$;

(ii) $x$ and $S x$ are fixed point of $S^{2}$ in $W$ and $V$, respectively.

Proof. By Theorem 19, there exists $x \in W$ such that $d(x, S x)=$ $d(W, V)$ and it follows that

$$
\begin{aligned}
d(W, V) \leq & d\left(S x, S^{2} x\right) \\
\leq & \varphi(d(x, S x)) d(x, S x) \\
& +(1-\varphi(d(x, S x))) d(W, V)
\end{aligned}
$$

$$
\begin{aligned}
& +L \min \left\{d\left(x, S^{2} x\right), d(S x, S x)\right\} \\
= & \varphi(d(x, S x)) d(x, S x) \\
& +(1-\varphi(d(x, S x))) d(W, V) \\
= & d(W, V) .
\end{aligned}
$$

Hence $d\left(S x, S^{2} x\right)=d(W, V)$. In the semisharp proximality of $(W, V)$, we have $S^{2} x=x$. Consider

$$
\begin{aligned}
d\left(x, S^{2}(S x)\right) & =d\left(x, S\left(S^{2} x\right)\right)=d(x, S x) \\
& =d(W, V)
\end{aligned}
$$

which implies that $S^{2}(S x)=S x$. Therefore, $x$ and $S x$ are fixed points of $S^{2}$ in $W$ and $V$, respectively. result.

Using the proof of Theorem 22, we obtain the following

Theorem 23. Let $W$ and $V$ be nonempty subsets of a metric space $(X, d)$ such that $(W, V)$ is a semisharp proximal pair, and $S: W \cup V \longrightarrow W \cup V$ is a Berinde MT-cyclic contraction with respect to $\varphi$. Let $x_{0} \in W$ be given and define a sequence $\left\{x_{n}\right\}$ in $W \cup V$ by $x_{n+1}=S x_{n}$, for all $n \geq 0$. If $\left\{x_{2 n+1}\right\}$ has a convergent subsequence in $V$, then the following hold:

(i) there exists $x \in V$ such that $d(x, S x)=d(W, V)$;

(ii) $x$ and $S x$ are fixed point of $S^{2}$ in $V$ and $W$, respectively.

By using Theorem 8 , we have the following corollary.

Corollary 24. Let $W$ and $V$ be nonempty complete subsets of a metric space $(X, d)$ such that $(W, V)$ satisfies the property $U C$, and $S: W \cup V \longrightarrow W \cup V$ is a Berinde MT-cyclic contraction with respect to $\varphi$. Let $x_{0} \in W$ be given and define a sequence $\left\{x_{n}\right\}$ in $W \cup V$ by $x_{n+1}=S x_{n}$, for all $n \geq 0$. Then the following hold:

(i) there exists $x \in W$ such that $d(x, S x)=d(W, V)$;

(ii) $x$ and $S x$ are fixed points of $S^{2}$ in $W$ and $V$, respectively.

We have discussed that, under some specific conditions, a Berinde MT-cyclic contraction with respect to $\varphi$ can be reduced to a cyclic contraction or an MT-cyclic contraction with respect to $\varphi$. Thus, Theorems 17 and 19 are generalizations of the results proved by Eldred and Veeramani [14] and Du and Lakzian [15], respectively. Hence, the three following corollaries are obtained directly from those theorems.

Corollary 25 (Eldred and Veeramani [14, Proposition 3.2]). Let $W$ and $V$ be nonempty closed subsets of a complete metric space $X$. Let $S: W \cup V \longrightarrow W \cup V$ be a cyclic contraction, $x_{0} \in W$, and define $x_{n+1}=S x_{n}$ for all $n \geq 0$. Suppose that $\left\{x_{2 n}\right\}$ has a convergent subsequence in $W$. Then there exists $x \in W$ such that $d(x, S x)=d(A, B)$.

Corollary 26 (Du and Lakzian [15, Theorem 2.3]). Let $W$ and $V$ be nonempty subsets of a metric space $(X, d)$ and $S: W \cup$ 
$V \rightarrow W \cup V$ be a cyclic mapping. Let $x_{0} \in W$ be given and define a sequence $\left\{x_{n}\right\}$ in $W \cup V$ by $x_{n+1}=S x_{n}$, for all $n \geq 0$. Suppose that the following conditions hold:

(i) $d(S x, S y) \leq d(x, y)$ for all $x \in W$ and $y \in V$;

(ii) $\left\{x_{2 n}\right\}$ has a convergent subsequence in $W$;

(iii) $\lim _{n \rightarrow \infty} d\left(x_{n}, x_{n+1}\right)=d(W, V)$.

Then there exists $x \in W$ such that $d(x, S x)=d(W, V)$.

Corollary 27 (Du and Lakzian [15, Theorem 2.4]). Let $W$ and $V$ be nonempty subsets of a metric space $(X, d)$ and $S$ : $W \cup V \longrightarrow W \cup V$ be a MT-cyclic contraction with respect to $\varphi$. Let $x_{0} \in W$ be given and define a sequence $\left\{x_{n}\right\}$ in $W \cup V$ by $x_{n+1}=\mathrm{S} x_{n}$, for all $n \geq 0$. Suppose that $\left\{x_{2 n}\right\}$ has a convergent subsequence in $W$. Then there exists $x \in W$ such that $d(x, S x)=d(W, V)$.

\section{Data Availability}

No data were used to support this study.

\section{Conflicts of Interest}

The authors declare that there are no conflicts of interest regarding the publication of this paper.

\section{Acknowledgments}

The authors would like to thank Professor Dr. Suthep Suantai for his helpful advice. This research is supported by Center of Excellence in Mathematics and Applied Mathematics, Chiang Mai University, and Faculty of Science, Chiang Mai University, Chiang Mai, Thailand.

\section{References}

[1] S. Banach, "Sur les opérations dans les ensembles abstraits et leur application aux équations intégrales," Fundamenta Mathematicae, vol. 3, pp. 133-181, 1922.

[2] V. Berinde, "Approximating fixed points of weak contractions using the Picard iteration," Nonlinear Analysis Forum , vol. 9, no. 1, pp. 43-53, 2004.

[3] K. Fan, "Extensions of two fixed point theorems of F. E. Browder," Mathematische Zeitschrift, vol. 112, pp. 234-240, 1969.

[4] S. Reich, "Approximate selections, best approximations, fixed points, and invariant sets," Journal of Mathematical Analysis and Applications, vol. 62, no. 1, pp. 104-113, 1978.

[5] V. M. Sehgal and S. P. Singh, "A theorem on best approximations," Numerical Functional Analysis and Optimization, vol. 10, no. 1-2, pp. 181-184, 1989.

[6] V. Vetrivel, P. Veeramani, and P. Bhattacharyya, "Some extensions of Fan's best approximation theorem," Numerical Functional Analysis and Optimization, vol. 13, no. 3-4, pp. 397-402, 1992.

[7] J. Anuradha and P. Veeramani, "Proximal pointwise contraction," Topology and its Applications, vol. 156, no. 18, pp. 29422948, 2009.
[8] S. Sadiq Basha, "Best proximity points: optimal solutions," Journal of Optimization Theory and Applications, vol. 151, no. 1, pp. 210-216, 2011.

[9] S. Sadiq Basha, "Best proximity point theorems," Journal of Approximation Theory, vol. 163, no. 11, pp. 1772-1781, 2011.

[10] W. A. Kirk, S. Reich, and P. Veeramani, "Proximinal retracts and best proximity pair theorems," Numerical Functional Analysis and Optimization, vol. 24, no. 7-8, pp. 851-862, 2003.

[11] V.S. Raj, "A best proximity point theorem for weakly contractive non-self-mappings," Nonlinear Analysis: Theory, Methods \& Applications, vol. 74, no. 14, pp. 4804-4808, 2011.

[12] M. Gabeleh, "Global optimal solutions of non-self mappings," "Politehnica" University of Bucharest. Scientific Bulletin. Series A. Applied Mathematics and Physics, vol. 75, no. 3, pp. 67-74, 2013.

[13] A. Abkar and M. Gabeleh, "Best proximity points of non-self mappings," TOP, vol. 21, no. 2, pp. 287-295, 2013.

[14] A. A. Eldred and P. Veeramani, "Existence and convergence of best proximity points," Journal of Mathematical Analysis and Applications, vol. 323, no. 2, pp. 1001-1006, 2006.

[15] W.-S. Du and H. Lakzian, "Nonlinear conditions for the existence of best proximity points," Journal of Inequalities and Applications, vol. 2012, article 206, 2012.

[16] H. Aydi, A. Felhi, and E. Karapinar, "On common best proximity points for generalized $\alpha$ - $\psi$-proximal contractions," Journal of Nonlinear Sciences and Applications. JNSA, vol. 9, no. 5, pp. 2658-2670, 2016.

[17] A. Felhi, "A note on "Convergence and best proximity points for Berinde's cyclic contraction with proximally complete property", Mathematical Methods in the Applied Sciences, vol. 41, no. 1, pp. 140-143, 2018.

[18] A. Felhi, "New best proximity results on partial ordered metric spaces," Journal of Advanced Mathematical Studies, vol. 10, no. 2, pp. 216-230, 2017.

[19] A. Felhi and H. Aydi, "Best proximity points and stability results for controlled proximal contractive set valued mappings," Fixed Point Theory and Applications, Paper No. 22, 23 pages, 2016.

[20] S. Reich, "Fixed points of contractive functions," Bollettino della Unione Matematica Italiana, vol. 5, pp. 26-42, 1972.

[21] N. Mizoguchi and W. Takahashi, "Fixed point theorems for multivalued mappings on complete metric spaces," Journal of Mathematical Analysis and Applications, vol. 141, no. 1, pp. 177$188,1989$.

[22] W. Du, "Some new results and generalizations in metric fixed point theory," Nonlinear Analysis: Theory, Methods \& Applications, vol. 73, no. 5, pp. 1439-1446, 2010.

[23] W. Du, "On coincidence point and fixed point theorems for nonlinear multivalued maps," Topology and its Applications, vol. 159, no. 1, pp. 49-56, 2012.

[24] T. Suzuki, M. Kikkawa, and C. Vetro, "The existence of best proximity points in metric spaces with the property UC," Nonlinear Analysis: Theory, Methods \& Applications, vol. 71, no. 7-8, pp. 2918-2926, 2009.

[25] G. S. Kosuru and P. Veeramani, "A note on existence and convergence of best proximity points for pointwise cyclic contractions," Numerical Functional Analysis and Optimization, vol. 32, no. 7, pp. 821-830, 2011.

[26] R. Espinola, G. S. Kosuru, and P. Veeramani, "Pythagorean property and best-proximity point theorems," Journal of Optimization Theory and Applications, vol. 164, no. 2, pp. 534-550, 2015. 


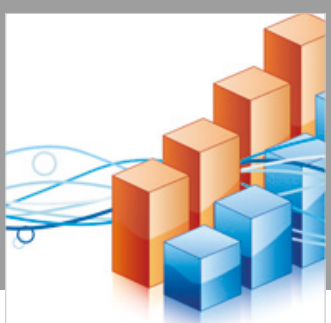

Advances in

Operations Research

\section{-n-m}
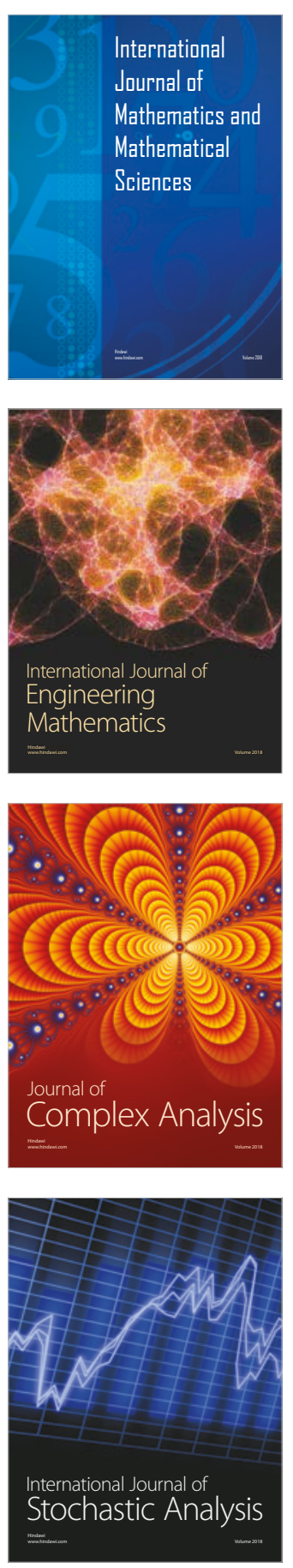
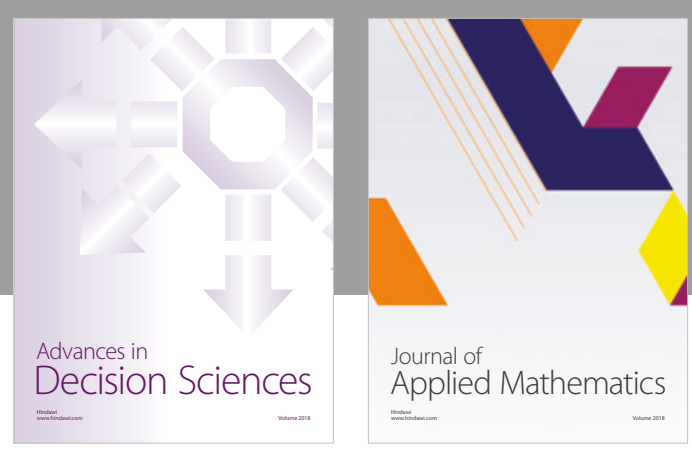

Journal of

Applied Mathematics
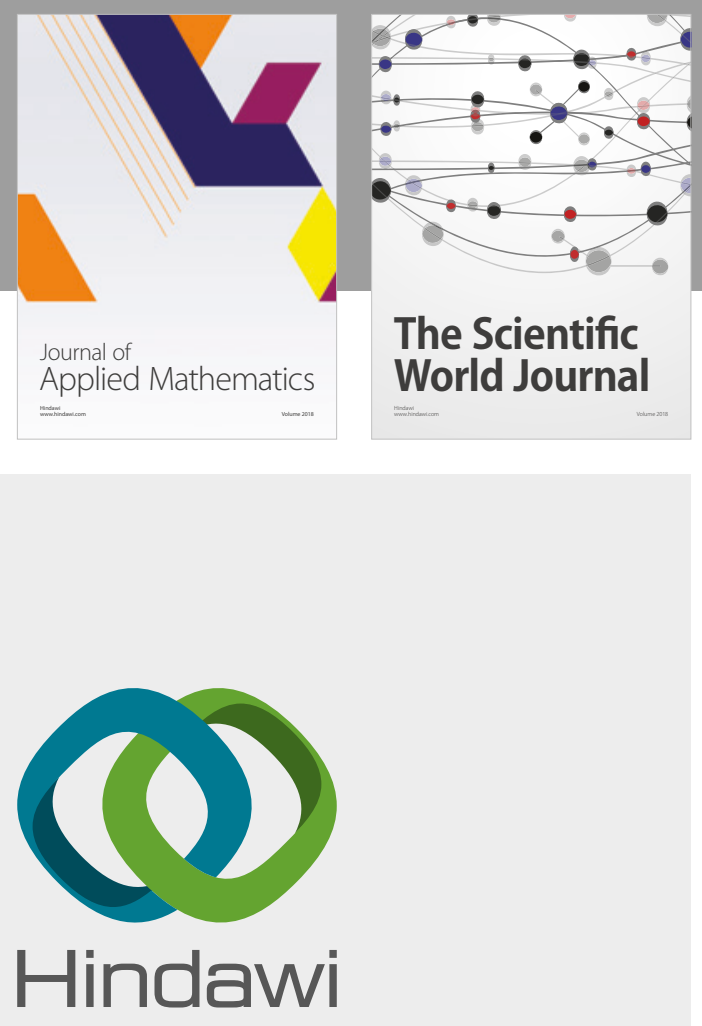

Submit your manuscripts at

www.hindawi.com

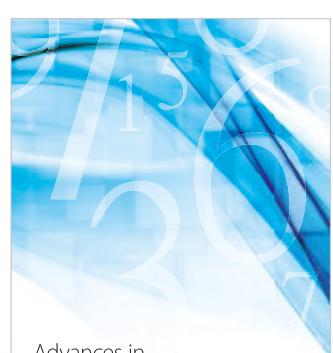

Advances in
Numerical Analysis
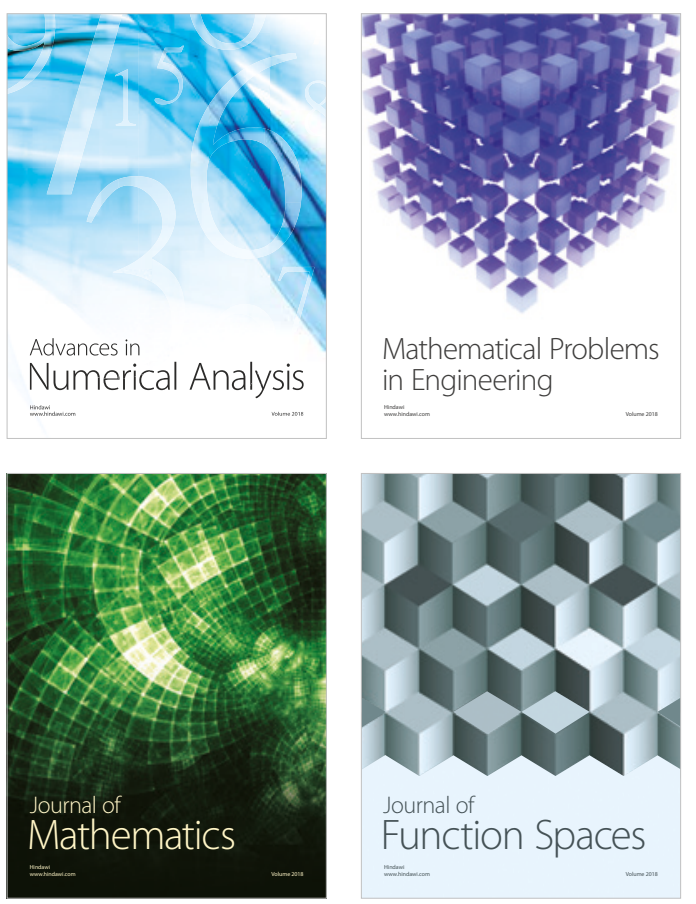

Mathematical Problems in Engineering

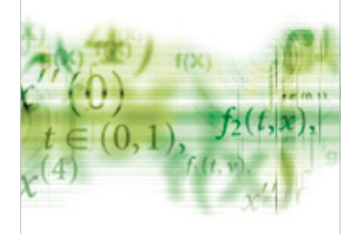

International Journal of

Differential Equations

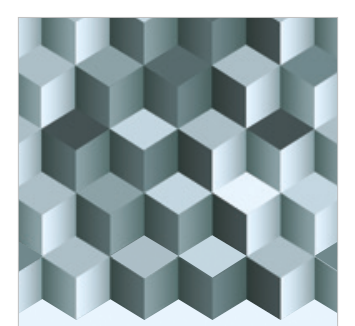

Journal of

Function Spaces

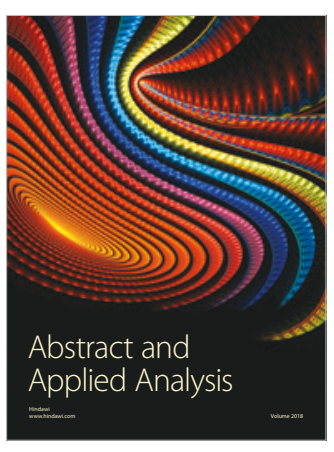

The Scientific

World Journal

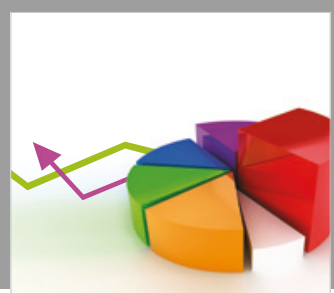

Journal of

Probability and Statistics
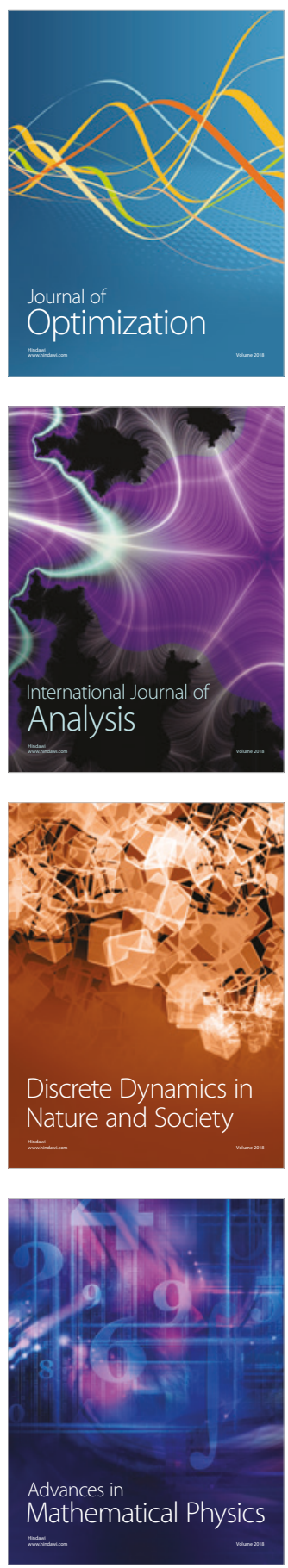